\title{
NORM INEQUALITIES IN MULTIDIMENSIONAL LORENTZ SPACES
}

\author{
BORIS SIMONOV and SERGEY TIKHONOV
}

\begin{abstract}
In this paper we obtain necessary and sufficient conditions for double trigonometric series to belong to generalized Lorentz spaces, not symmetric in general. Estimates for the norms are given in terms of coefficients.
\end{abstract}

\section{Introduction}

Let $f(x, y)$ be a measurable function on $[0,2 \pi]^{2}$. We define the rearrangement of $f(x, y)$ with respect to $y$ to be $f_{y}^{*}\left(x, t_{2}\right)=(f(x, y))_{y}^{*}\left(x, t_{2}\right)$, i.e., $f_{y}^{*}\left(x, t_{2}\right)$ is a non-increasing function on $t_{2}$ and the functions $f_{y}^{*}\left(x, t_{2}\right)$ and $|f(x, y)|$ are equimeasurable as functions of one variable for almost all $x$. We define the rearrangement of $f_{y}^{*}\left(x, t_{2}\right)$ with respect to $x$ to be $f_{y x}^{*}\left(t_{1}, t_{2}\right)$. Therefore $f_{y x}^{*}\left(t_{1}, t_{2}\right)$ is non-increasing on $t_{1}$ and $t_{2}$ and equimeasurable with $|f(x, y)|$.

According to [1]-[2], if $0<\alpha<\infty$, we say that a measurable function $f(x, y)$, which is $2 \pi$-periodic on each variable, belongs to the two-dimensional weighted Lorentz space $\Lambda_{2}^{\alpha}(\omega)$, if

$$
\|f\|_{\Lambda_{2}^{\alpha}(\omega)}=\left(\int_{0}^{2 \pi} \int_{0}^{2 \pi} w\left(t_{1}, t_{2}\right)\left(f_{y x}^{*}\left(t_{1}, t_{2}\right)\right)^{\alpha} d t_{1} d t_{2}\right)^{\frac{1}{\alpha}}<\infty,
$$

where the weighted function $w \in W$, i.e., $w\left(t_{1}, t_{2}\right)$ is an a.e. positive locally integrable function on $[0,2 \pi]^{2}$.

Similarly, we say that $f(x, y)$ belongs to $\bar{\Lambda}_{2}^{\alpha}(\omega)$ provided that

$$
\|f\|_{\bar{\Lambda}_{2}^{\alpha}(\omega)}=\left(\int_{0}^{2 \pi} \int_{0}^{2 \pi} w\left(t_{1}, t_{2}\right)\left(f_{x y}^{*}\left(t_{1}, t_{2}\right)\right)^{\alpha} d t_{1} d t_{2}\right)^{\frac{1}{\alpha}}<\infty .
$$

We consider the following series

$$
\sum_{m=0}^{\infty} \sum_{n=0}^{\infty} a_{m n} \cos m x \cos n y,
$$

Received April 18, 2007. 


$$
\begin{aligned}
& \sum_{m=0}^{\infty} \sum_{n=1}^{\infty} a_{m n} \cos m x \sin n y, \\
& \sum_{m=1}^{\infty} \sum_{n=0}^{\infty} a_{m n} \sin m x \cos n y, \\
& \sum_{m=1}^{\infty} \sum_{n=1}^{\infty} a_{m n} \sin m x \sin n y,
\end{aligned}
$$

where the coefficients $a_{m n}$ are real numbers and $\cos 0 x:=\cos 0 y:=\frac{1}{2}$. We also suppose that the sequence $\left\{a_{m n}\right\}$ satisfies the condition

$$
a_{m n} \rightarrow 0 \quad \text { as } \quad m+n \rightarrow \infty \text {. }
$$

For integers $k_{1}$ and $k_{2}$ we define

$$
\Delta_{k_{1} k_{2}} a_{m n}=\sum_{i=0}^{k_{1}}(-1)^{i} C_{k_{1}}^{i} \sum_{j=0}^{k_{2}}(-1)^{j} C_{k_{2}}^{j} a_{m+i n+j} \quad(m, n \geq 0)
$$

where $C_{k}^{l}=\frac{k(k-1) \cdots(k-l+1)}{l !}$ for $l \geq 1$ and $C_{k}^{l}=1$ for $l=0$.

We note that if $\left\{a_{m n}\right\}$ satisfies (1.5) and $\Delta_{k_{1} k_{2}} a_{m n} \geq 0$ for integers $k_{1}, k_{2} \geq 1$, then $\Delta_{s_{1} s_{2}} a_{m n} \geq 0$ for all $0 \leq s_{1} \leq k_{1}$ and $0 \leq s_{2} \leq k_{2}$.

By $C, C_{i}$ we denote positive constants that may be different on different occasions. Also, $F \asymp G$ means that there exist constants $C_{1}$ and $C_{2}$ such that $C_{1} F \leq G \leq C_{2} F$.

Now we recall the definition of convergence of double series in the Pringsheim's sense. The partial sums of the series $\sum_{\mu, \nu=0}^{\infty} c_{\mu \nu}$ are defined to be $S_{m n}=\sum_{\nu=0}^{n} \sum_{\mu=0}^{m} c_{\mu \nu}$. If there exists a number $S$ such that for any $\varepsilon>0$ there exist integers $k$ and $l$ such that $\left|S_{m n}-S\right|<\varepsilon$ for all $n>k$ and $m>l$, then series $\sum_{\mu, \nu} c_{\mu \nu}$ is said to converge to $S$, in the sense of Pringsheim.

It is well-known (see e.g. [7]) that if a sequence $\left\{a_{m n}\right\}$ satisfies (1.5) and $\Delta_{k_{1} k_{2}} a_{m n} \geq 0$ for any integers $k_{1}$ and $k_{2}$, then series (1.1)-(1.4) converge in Pringsheim's sense except, possibly, on a set of measure zero. We define by $f_{1}(x, y), f_{2}(x, y), f_{3}(x, y)$, and $f_{4}(x, y)$ the sums of series (1.1)-(1.4), respectively.

The aim of the paper is to study necessary and sufficient conditions for the functions $f_{1}(x, y), f_{2}(x, y), f_{3}(x, y)$, and $f_{4}(x, y)$ to belong to the Lorentz spaces, not symmetric in general, in terms of coefficients $\left\{a_{m n}\right\}$.

This problem has a long history starting from the well-known theorem by Hardy and Littlewood (see [8], [17, V.2, XII, §6]): A necessary and sufficient 
condition that the function

$$
f(x) \sim \sum_{n}\left(a_{n} \cos n x+b_{n} \sin n x\right), \quad a_{n}, b_{n} \downarrow
$$

should belong to $L^{p}, 1<p<\infty$, is that

$$
\sum_{n}\left(a_{n}^{p}+b_{n}^{p}\right) n^{p-2}<\infty
$$

This result was generalized by Sagher for the Lorentz spaces (see [14] and [15]): Assume that $f(x)$ is either the Fourier sine or Fourier cosine series associated with $\left\{a_{n}\right\}$. If $a_{n} \downarrow$, then for $1<p<\infty$ and $0<q \leq \infty$,

$$
\|f(x)\|_{L^{p, q}} \asymp\left\|\left\{a_{n}\right\}\right\|_{l^{p^{\prime}, q}}
$$

where $L^{p, q}$ and $l^{p^{\prime}, q}$ are continuous and discrete Lorentz spaces [3].

For non-weighted multidimensional $L^{p}$-spaces, where $1<p<\infty$, the Hardy-Littlewood-type result was obtained by Moricz [12]. The cases of different Lebesgue and Lorentz spaces were also investigated in [4], [6], [11], [13], [16], among others.

In section 2 we present our main results for the spaces $\Lambda_{2}^{\alpha}(\omega)$ and $\bar{\Lambda}_{2}^{\alpha}(\omega)$ in the case of $\alpha \in(0, \infty)$. First (Theorem 2.1), we find the estimate of $\left\|f_{i}\right\|_{\Lambda_{2}^{\alpha}(\omega)}$ from above. Next (Theorems 2.2 and 2.3), we give the necessary conditions for $f_{i}$ to belong to $\Lambda_{2}^{\alpha}(\omega)$, depending on the monotonicity properties of the weight $\omega$. Sections 3 and 4 contain the preliminary results and proofs, respectively.

Finally, we remark that our results, in particular, provide criteria for $f_{1}, f_{2}$, $f_{3}$, and $f_{4}$ to belong to classical Lorentz space $L^{p, q}, 0<p, q<\infty$, and to the Lorentz-Zygmund space $L^{p, q} \varphi(L)$ (here $\varphi$ is a slowly varying function; see [5], [11]).

\section{Main Results}

For convenience purposes we set

$$
w_{m n}:=\int_{\pi /(n+1)}^{\pi / \bar{n}} \int_{\pi /(m+1)}^{\pi / \bar{m}} w(x, y) d x d y, \quad \text { where } \bar{n}:= \begin{cases}\frac{1}{2} & \text { if } n=0, \\ n & \text { if } n \in \mathrm{N} .\end{cases}
$$

Then our results are read as follows.

THEOREM 2.1. Let $0<\alpha<\infty, w \in W$, and let $w$ satisfy the following condition: for all $\delta_{1}, \delta_{2} \in(0,2 \pi)$

$$
\int_{0}^{\delta_{1}} \int_{0}^{\delta_{2}} w\left(t_{1}, t_{2}\right) d t_{1} d t_{2} \leq C \int_{\frac{\delta_{1}}{2}}^{\delta_{1}} \int_{\frac{\delta_{2}}{2}}^{\delta_{2}} w\left(t_{1}, t_{2}\right) d t_{1} d t_{2}
$$


where $C$ is independent of $\delta_{1}, \delta_{2}$. Suppose the sequence $\left\{a_{m n}\right\}$ satisfies condition (1.5) and $\Delta_{k_{1} k_{2}} a_{m n} \geq 0$ for all $m, n \in \mathrm{N}$ and appropriate $k_{1}, k_{2}$ appearing in $(\mathrm{A})-(\mathrm{D})$.

(A) If $k_{1}=2$ and $k_{2}=2$, then

(2.2) $\left\|f_{1}(x, y)\right\|_{\Lambda_{2}^{\alpha}(\omega)} \leq C_{1}\left(\sum_{n=0}^{\infty}(n+1)^{2 \alpha} \sum_{m=0}^{\infty}(m+1)^{2 \alpha} w_{m n}\left(\Delta_{11} a_{m n}\right)^{\alpha}\right)^{\frac{1}{\alpha}}$;

(B) If $k_{1}=2$ and $k_{2}=1$, then

(2.3) $\left\|f_{2}(x, y)\right\|_{\Lambda_{2}^{\alpha}(\omega)} \leq C_{2}\left(\sum_{n=1}^{\infty}(n+1)^{\alpha} \sum_{m=0}^{\infty}(m+1)^{2 \alpha} w_{m n}\left(\Delta_{10} a_{m n}\right)^{\alpha}\right)^{\frac{1}{\alpha}}$;

(C) If $k_{1}=1$ and $k_{2}=2$, then

$$
\left\|f_{3}(x, y)\right\|_{\Lambda_{2}^{\alpha}(\omega)} \leq C_{3}\left(\sum_{n=0}^{\infty}(n+1)^{2 \alpha} \sum_{m=1}^{\infty}(m+1)^{\alpha} w_{m n}\left(\Delta_{01} a_{m n}\right)^{\alpha}\right)^{\frac{1}{\alpha}}
$$

(D) If $k_{1}=1$ and $k_{2}=1$, then

$$
\left\|f_{4}(x, y)\right\|_{\Lambda_{2}^{\alpha}(\omega)} \leq C_{4}\left(\sum_{n=1}^{\infty}(n+1)^{\alpha} \sum_{m=1}^{\infty}(m+1)^{\alpha} w_{m n} a_{m n}^{\alpha}\right)^{\frac{1}{\alpha}} ;
$$

where $C_{1}, C_{2}, C_{3}, C_{4}$ are independent of $\left\{a_{m n}\right\}$.

Remark 2.1. For any $\gamma_{1}, \gamma_{2}>0$ and for any slowly varying functions $\varphi_{1}(x), \varphi_{2}(y)$ on $(0, \infty), w\left(t_{1}, t_{2}\right)=t_{1}^{\gamma_{1}-1} \varphi_{1}\left(1 / t_{1}\right) t_{2}^{\gamma_{2}-1} \varphi_{1}\left(1 / t_{2}\right)$ satisfies $(2.1)$.

Theorem 2.2. Let $0<\alpha<\infty, w\left(t_{1}, t_{2}\right) \in W$, and let $w\left(t_{1}, t_{2}\right)$ be nonincreasing with respect to $t_{1}$ for almost all $t_{2}$ and non-increasing with respect to $t_{2}$ for almost all $t_{1}$. Suppose the sequence $\left\{a_{m n}\right\}$ satisfies condition (1.5) and $\Delta_{k_{1} k_{2}} a_{m n} \geq 0$ for all $m, n \in \mathrm{N}$ and appropriate $k_{1}, k_{2}$ appearing in (A)-(D).

(A) If $k_{1}=2$ and $k_{2}=2$, then

(2.6) $\left\|f_{1}(x, y)\right\|_{\Lambda_{2}^{\alpha}(\omega)} \geq C_{1}\left(\sum_{n=0}^{\infty}(n+1)^{2 \alpha} \sum_{m=0}^{\infty}(m+1)^{2 \alpha} w_{m n}\left(\Delta_{11} a_{m n}\right)^{\alpha}\right)^{\frac{1}{\alpha}}$;

(B) If $k_{1}=2$ and $k_{2}=1$, then

(2.7) $\left\|f_{2}(x, y)\right\|_{\Lambda_{2}^{\alpha}(\omega)} \geq C_{2}\left(\sum_{n=1}^{\infty}(n+1)^{\alpha} \sum_{m=0}^{\infty}(m+1)^{2 \alpha} w_{m n}\left(\Delta_{10} a_{m n}\right)^{\alpha}\right)^{\frac{1}{\alpha}}$; 
(C) If $k_{1}=1$ and $k_{2}=2$, then

(2.8) $\left\|f_{3}(x, y)\right\|_{\Lambda_{2}^{\alpha}(\omega)} \geq C_{3}\left(\sum_{n=0}^{\infty}(n+1)^{2 \alpha} \sum_{m=1}^{\infty}(m+1)^{\alpha} w_{m n}\left(\Delta_{01} a_{m n}\right)^{\alpha}\right)^{\frac{1}{\alpha}}$;

(D) If $k_{1}=1$ and $k_{2}=1$, then

$$
\left\|f_{4}(x, y)\right\|_{\Lambda_{2}^{\alpha}(\omega)} \geq C_{4}\left(\sum_{n=1}^{\infty}(n+1)^{\alpha} \sum_{m=1}^{\infty}(m+1)^{\alpha} w_{m n} a_{m n}^{\alpha}\right)^{\frac{1}{\alpha}} ;
$$

where $C_{1}, C_{2}, C_{3}, C_{4}$ are independent of $\left\{a_{m n}\right\}$.

Theorem 2.3. Let $1 \leq \alpha<\infty, w\left(t_{1}, t_{2}\right) \in W$, and let $w\left(t_{1}, t_{2}\right)$ satisfy the following two conditions:

(1) for almost all $t_{2} \in[0,2 \pi] w\left(t_{1}, t_{2}\right)$ is non-decreasing and satisfies $\Delta_{2}$ condition with respect to $t_{1}$, i.e., $w\left(2 t_{1}, t_{2}\right) \leq C w\left(t_{1}, t_{2}\right)$;

(2) for almost all $t_{1} \in[0,2 \pi], w\left(t_{1}, t_{2}\right)$ is non-decreasing and satisfies $\Delta_{2}$-condition with respect to $t_{2}$, i.e., $w\left(t_{1}, 2 t_{2}\right) \leq C w\left(t_{1}, t_{2}\right)$.

Suppose the sequence $\left\{a_{m n}\right\}$ satisfies condition (1.5) and $\Delta_{k_{1} k_{2}} a_{m n} \geq 0$ for all $m, n \in \mathrm{N}$ and appropriate $k_{1}, k_{2}$ appearing in (A)-(D).

(A) If $k_{1}=2$ and $k_{2}=2$, then inequality (2.6) is satisfied;

(B) If $k_{1}=2$ and $k_{2}=1$, then inequality (2.7) is satisfied;

(C) If $k_{1}=1$ and $k_{2}=2$, then inequality (2.8) is satisfied;

(D) If $k_{1}=1$ and $k_{2}=1$, then inequality (2.9) is satisfied;

where $C_{1}, C_{2}, C_{3}, C_{4}$ are independent of $\left\{a_{m n}\right\}$.

COROLlary 2.1. Under the conditions of Theorem 2.1 and Theorem 2.2 (or Theorem 2.3), we have

$$
\left\|f_{j}(x, y)\right\|_{\Lambda_{2}^{\alpha}(\omega)} \asymp\left\|f_{j}(x, y)\right\|_{\bar{\Lambda}_{2}^{\alpha}(\omega)}, \quad j=1,2,3,4 .
$$

We note that, in general, (2.10) does not hold (see [2] and [5]). We also remark that in $[2, \S 3]$ the authors showed that if $\|\cdot\|_{\Lambda_{2}^{p}(\omega)}$ is a rearrangement invariant norm (see [3, Ch. 2]), then $\omega$ is a constant and $\Lambda_{2}^{p}(\omega) \equiv L^{p}\left([0,2 \pi]^{2}\right)$. The following corollary follows from Theorems 2.1-2.3 (see also[16]).

Corollary 2.2. Let $0<p<\infty$. Suppose the sequence $\left\{a_{m n}\right\}$ satisfies condition (1.5) and $\Delta_{k_{1} k_{2}} a_{m n} \geq 0$ for all $m, n \in \mathrm{N}$ and appropriate $k_{1}, k_{2}$ appearing in $(\mathrm{A})-(\mathrm{D})$. 
(A) If $k_{1}=2$ and $k_{2}=2$, then

$$
\left\|f_{1}(x, y)\right\|_{L^{p}\left([0,2 \pi]^{2}\right)} \asymp\left(\sum_{n=0}^{\infty}(n+1)^{2 p-2} \sum_{m=0}^{\infty}(m+1)^{2 p-2}\left(\Delta_{11} a_{m n}\right)^{p}\right)^{\frac{1}{p}} ;
$$

(B) If $k_{1}=2$ and $k_{2}=1$, then

$$
\left\|f_{2}(x, y)\right\|_{L^{p}\left([0,2 \pi]^{2}\right)} \asymp\left(\sum_{n=1}^{\infty}(n+1)^{p-2} \sum_{m=0}^{\infty}(m+1)^{2 p-2}\left(\Delta_{10} a_{m n}\right)^{p}\right)^{\frac{1}{p}} ;
$$

(C) If $k_{1}=1$ and $k_{2}=2$, then

$$
\left\|f_{3}(x, y)\right\|_{L^{p}\left([0,2 \pi]^{2}\right)} \asymp\left(\sum_{n=0}^{\infty}(n+1)^{2 p-2} \sum_{m=1}^{\infty}(m+1)^{p-2}\left(\Delta_{01} a_{m n}\right)^{p}\right)^{\frac{1}{p}} ;
$$

(D) If $k_{1}=1$ and $k_{2}=1$, then

$$
\left\|f_{4}(x, y)\right\|_{L^{p}\left([0,2 \pi]^{2}\right)} \asymp\left(\sum_{n=1}^{\infty}(n+1)^{p-2} \sum_{m=1}^{\infty}(m+1)^{p-2} a_{m n}^{p}\right)^{\frac{1}{p}} .
$$

This result is the two-dimensional version of the classical Hardy-Littlewood theorem. Note also that if $1<p<\infty$ and $k_{1}=k_{2}=1$, then (compare with [12])

$\left\|f_{j}(x, y)\right\|_{L^{p}\left([0,2 \pi]^{2}\right)}$

$$
\asymp\left(\sum_{n=1}^{\infty}(n+1)^{p-2} \sum_{m=1}^{\infty}(m+1)^{p-2} a_{m n}^{p}\right)^{\frac{1}{p}}, \quad j=1,2,3,4 .
$$

\section{Lemmas}

Let

$$
\begin{aligned}
& B_{0}^{1}(x)=\frac{1}{2} \\
& B_{n}^{1}(x)=\frac{1}{2}+\cos x+\cdots+\cos n x \quad \text { for } \quad n \geq 1 \\
& B_{n}^{k}(x)=\sum_{m=0}^{n} B_{m}^{k-1}(x) \quad \text { for } \quad k=2,3, \ldots \text { and } n=0,1,2, \ldots ;
\end{aligned}
$$




$$
\begin{aligned}
& \bar{B}_{n}^{1}(x)=\sin x+\cdots+\sin n x \quad \text { for } \quad n=1,2, \ldots \\
& \bar{B}_{n}^{k}(x)=\sum_{m=1}^{n} \bar{B}_{m}^{k-1}(x) \quad \text { for } \quad k=2,3, \ldots \text { and } n=1,2, \ldots
\end{aligned}
$$

We consider series

$$
\begin{aligned}
& \sum_{n=0}^{\infty} \sum_{m=0}^{\infty} \Delta_{k_{1} k_{2}} a_{m n} B_{m}^{k_{1}}(x) B_{n}^{k_{2}}(y) ; \\
& \sum_{n=1}^{\infty} \sum_{m=0}^{\infty} \Delta_{k_{1} k_{2}} a_{m n} B_{m}^{k_{1}}(x) \bar{B}_{n}^{k_{2}}(y) ; \\
& \sum_{n=0}^{\infty} \sum_{m=1}^{\infty} \Delta_{k_{1} k_{2}} a_{m n} \bar{B}_{m}^{k_{1}}(x) B_{n}^{k_{2}}(y) ; \\
& \sum_{n=1}^{\infty} \sum_{m=1}^{\infty} \Delta_{k_{1} k_{2}} a_{m n} \bar{B}_{m}^{k_{1}}(x) \bar{B}_{n}^{k_{2}}(y) .
\end{aligned}
$$

Lemma 3.1. Suppose the sequence $\left\{a_{m n}\right\}$ satisfies condition (1.5) and $\Delta_{k_{1} k_{2}} a_{m n} \geq 0$ for integers $k_{1}$ and $k_{2}$. Then any series (3.1)-(3.4) converges in Pringsheim's sense except possibly the set of measure zero to $f_{1}(x, y)$, $f_{2}(x, y), f_{3}(x, y)$, and $f_{4}(x, y)$, respectively.

The proof follows easily from the Abel transform (see also [16]).

Lemma 3.2 ([10]). Let $a_{n}>0, b_{n} \geq 0(n=1,2, \ldots) ; 1 \leq p<\infty$. Then

$$
\begin{aligned}
& \sum_{k=1}^{\infty} a_{k}\left(\sum_{m=1}^{k} b_{m}\right)^{p} \leq p^{p} \sum_{m=1}^{\infty} a_{m}^{1-p}\left(b_{m} \sum_{n=m}^{\infty} a_{n}\right)^{p} ; \\
& \sum_{k=1}^{\infty} a_{k}\left(\sum_{m=k}^{\infty} b_{m}\right)^{p} \leq p^{p} \sum_{m=1}^{\infty} a_{m}^{1-p}\left(b_{m} \sum_{n=1}^{m} a_{n}\right)^{p} .
\end{aligned}
$$

Lemma 3.3 ([9, Ch. II, §2]). Let $f$ and $g$ be measurable functions on $[0,2 \pi]$. Then

$$
\begin{gathered}
(f+g)^{*}\left(t_{1}+t_{2}\right) \leq f^{*}\left(t_{1}\right)+g^{*}\left(t_{2}\right), \\
(f g)^{*}\left(t_{1}+t_{2}\right) \leq f^{*}\left(t_{1}\right) g^{*}\left(t_{2}\right) .
\end{gathered}
$$

Lemma 3.4 ([9, Ch. II, §2]). Let $f$ and $g$ be measurable functions on $[0,2 \pi]$. Suppose $|f(t)| \leq|g(t)|$; then $f^{*}(t) \leq g^{*}(t)$. 
Lemma 3.5 ([3, Ch. $2, \S 2])$. Let $f$ be a measurable function on $[0,2 \pi]$. Then for all $0<\alpha<\infty$

$$
\left(f^{*}(t)\right)^{\alpha}=\left(|f|^{\alpha}\right)^{*}(t)
$$

Lemma 3.6 ([9]). Let $h(t)$ be an increasing non-negative function. Then

$$
\int_{0}^{\infty}(f+g)^{*}(t) h(t) d t \geq \int_{0}^{\infty} f^{*}(t) h(t) d t+\int_{0}^{\infty} g^{*}(t) h(t) d t .
$$

Lemma 3.7 ([5]). (A) Suppose $|f(x, y)| \leq|g(x, y)|$; then $f_{y x}^{*}\left(t_{1}, t_{2}\right) \leq$ $g_{y x}^{*}\left(t_{1}, t_{2}\right)$;

(B) $(f+g)_{y x}^{*}\left(s_{1}+s_{2}, t_{1}+t_{2}\right) \leq f_{y x}^{*}\left(s_{1}, t_{1}\right)+g_{y x}^{*}\left(s_{2}, t_{2}\right)$.

Lemma 3.8 ([9, Ch. II, §2]). Let $f(t)$ and $g(t)$ be non-negative summable functions on $[0,2 \pi]$ such that

$$
\int_{0}^{x} f(t) d t \leq \int_{0}^{x} g(t) d t
$$

Suppose $h(t)$ is non-increasing non-negative function on $(0,2 \pi)$; then

$$
\int_{0}^{2 \pi} f(t) h(t) d t \leq \int_{0}^{2 \pi} g(t) h(t) d t .
$$

Lemma 3.9 ([5]). Let $f^{*}\left(t_{1}, t_{2}\right)=f_{y x}^{*}\left(t_{1}, t_{2}\right), \sigma>0$ and let

$$
\begin{aligned}
\lambda_{f}(\sigma) & =\mu\left\{(x, y) \in \Omega_{1} \times \Omega_{2}:|f(x, y)|>\sigma\right\}, \\
\lambda_{f^{*}}(\sigma) & =\mu\left\{(s, t) \in[0, \infty) \times[0, \infty): f^{*}(s, t)>\sigma\right\} .
\end{aligned}
$$

Then $\lambda_{f}(\sigma)=\lambda_{f^{*}}(\sigma)$.

Lemma 3.10. Let a measurable set $E \subset[0, a] \times[0, b]$, where $0<a, b<$ $\infty$. Suppose for some $k \in(0,1)$,

$$
\mu E>k \mu([0, a] \times[0, b])=k a b .
$$

Then

$$
\begin{aligned}
U:=\{(s, t) \in & {\left.\left[0, \frac{k a}{2}\right] \times\left[0, \frac{k b}{2}\right]\right\} } \\
& \subset\left\{(s, t) \in[0, \infty) \times[0, \infty):\left(\chi_{E}(x, y)\right)_{y x}^{*}(s, t)>\frac{1}{2}\right\}=: T .
\end{aligned}
$$


Proof. From the non-increase of $\left(\chi_{E}(x, y)\right)_{y x}^{*}(s, t)$ it is clear that the boundary of $\left\{(s, t):\left(\chi_{E}(x, y)\right)_{y x}^{*}(s, t)>\frac{1}{2}\right\}$ is the non-decreasing function $t=d(s)$. Let us assume that $U \nsubseteq T$. Therefore, $\mu(T) \leq \frac{k a b}{2}+\frac{k a b}{2}-\frac{k^{2} a b}{4}<$ $k a b$. By Lemma 3.9, we have $\mu E=\mu T$ and hence $\mu E<k a b$ which contradicts our assumption. Thus, $U \subset T$, which completes the proof.

\section{Proofs}

Proof of Theorem 2.1. By Lemma 3.1, the following identity holds a.e.

$$
F(x, y)=\sum_{n=0}^{\infty} \sum_{m=0}^{\infty} \Delta_{l_{1} l_{2}} a_{m n} \widetilde{B}_{m}^{l_{1}}(x) \widetilde{B}_{n}^{l_{2}}(y),
$$

where in the case of
(A) $F(x, y)=f_{1}(x, y), l_{1}=l_{2}=2, \widetilde{B}_{m}^{l_{1}}(x)=B_{m}^{2}(x), \widetilde{B}_{n}^{l_{2}}(y)=B_{n}^{2}(y)$;
(B) $F(x, y)=f_{2}(x, y), l_{1}=2, l_{2}=1, \widetilde{B}_{m}^{l_{1}}(x)=B_{m}^{2}(x), \widetilde{B}_{n}^{l_{2}}(y)=\bar{B}_{n}^{1}(y)$, and $\Delta_{21} a_{m 0}=\Delta_{10} a_{m 0}=0, m=0,1,2, \ldots$;
(C) $F(x, y)=f_{3}(x, y), l_{1}=1, l_{2}=2, \widetilde{B}_{m}^{l_{1}}(x)=\bar{B}_{m}^{1}(x), \widetilde{B}_{n}^{l_{2}}(y)=B_{n}^{2}(y)$, and $\Delta_{12} a_{0 n}=\Delta_{01} a_{0 n}=0, n=0,1,2, \ldots$;
(D) $F(x, y)=f_{4}(x, y), l_{1}=l_{2}=1, \widetilde{B}_{m}^{l_{1}}(x)=\bar{B}_{m}^{1}(x), \widetilde{B}_{n}^{l_{2}}(y)=\bar{B}_{n}^{1}(y)$, $0,1,2, \ldots$ and $\Delta_{11} a_{m 0}=a_{m 0}=0, m=0,1,2, \ldots, \Delta_{11} a_{0 n}=a_{0 n}=0, n=$

We estimate $\|F(x, y)\|_{\Lambda_{2}^{p}(\omega)}$ from above. Using Lemmas 3.3 and 3.4, we obtain

$$
\begin{aligned}
& \|F(x, y)\|_{\Lambda_{2}^{\alpha}(\omega)}^{\alpha} \\
& =\int_{0}^{2 \pi} \int_{0}^{2 \pi} \omega\left(t_{1}, t_{2}\right)\left(F ( x , y ) \left[\chi_{(0, \pi]}(x) \chi_{(0, \pi]}(y)+\chi_{(0, \pi]}(x) \chi_{(\pi, 2 \pi]}(y)\right.\right. \\
& \left.\left.\quad+\chi_{(\pi, 2 \pi]}(x) \chi_{(0, \pi]}(y)+\chi_{(\pi, 2 \pi]}(x) \chi_{(\pi, 2 \pi]}(y)\right]_{y x}^{*}\left(t_{1}, t_{2}\right)\right)^{\alpha} d t_{1} d t_{2} \\
& \leq C \int_{0}^{\pi / 2} \int_{0}^{\pi / 2} \omega\left(4 t_{1}, 4 t_{2}\right)\left(\left[F(x, y) \chi_{(0, \pi]}(x) \chi_{(0, \pi]}(y)\right]_{y x}^{*}\left(t_{1}, t_{2}\right)\right)^{\alpha} d t_{1} d t_{2} \\
& \leq C\left(I_{1}+I_{2}+I_{3}+I_{4}\right),
\end{aligned}
$$

where

$$
\begin{aligned}
I_{1}= & \sum_{n=0}^{\infty} \int_{\pi / 2^{n+4}}^{\pi / 2^{n+3}} \sum_{m=0}^{\infty} \int_{\pi / 2^{m+4}}^{\pi / 2^{m+3}} \omega\left(16 t_{1}, 16 t_{2}\right) \\
& \left(\left[\sum_{\nu=0}^{2^{n+1}} \sum_{\mu=0}^{2^{m+1}} \Delta_{l_{1} l_{2}} a_{\mu \nu} \widetilde{B}_{\mu}^{l_{1}}(x) \widetilde{B}_{\nu}^{l_{2}}(y) \chi_{(0, \pi]}(x) \chi_{(0, \pi]}(y)\right]_{y x}^{*}\left(t_{1}, t_{2}\right)\right)^{\alpha} d t_{1} d t_{2} ;
\end{aligned}
$$




$$
\begin{aligned}
& I_{2}=\sum_{n=0}^{\infty} \int_{\pi / 2^{n+4}}^{\pi / 2^{n+3}} \sum_{m=0}^{\infty} \int_{\pi / 2^{m+4}}^{\pi / 2^{m+3}} \omega\left(16 t_{1}, 16 t_{2}\right) \\
& \left(\left[\sum_{\nu=2^{n+1}+1}^{\infty} \sum_{\mu=0}^{2^{m+1}} \Delta_{l_{1} l_{2}} a_{\mu \nu} \widetilde{B}_{\mu}^{l_{1}}(x) \widetilde{B}_{\nu}^{l_{2}}(y) \chi_{(0, \pi]}(x) \chi_{(0, \pi]}(y)\right]_{y x}^{*}\left(t_{1}, t_{2}\right)\right)^{\alpha} d t_{1} d t_{2} ; \\
& I_{3}=\sum_{n=0}^{\infty} \int_{\pi / 2^{n+4}}^{\pi / 2^{n+3}} \sum_{m=0}^{\infty} \int_{\pi / 2^{m+4}}^{\pi / 2^{m+3}} \omega\left(16 t_{1}, 16 t_{2}\right) \\
& \left(\left[\sum_{\nu=0}^{2^{n+1}} \sum_{\mu=2^{m+1}+1}^{\infty} \Delta_{l_{1} l_{2}} a_{\mu \nu} \widetilde{B}_{\mu}^{l_{1}}(x) \widetilde{B}_{\nu}^{l_{2}}(y) \chi_{(0, \pi]}(x) \chi_{(0, \pi]}(y)\right]_{y x}^{*}\left(t_{1}, t_{2}\right)\right)^{\alpha} d t_{1} d t_{2} ; \\
& I_{4}=\sum_{n=0}^{\infty} \int_{\pi / 2^{n+4}}^{\pi / 2^{n+3}} \sum_{m=0}^{\infty} \int_{\pi / 2^{m+4}}^{\pi / 2^{m+3}} \omega\left(16 t_{1}, 16 t_{2}\right) \\
& \left(\left[\sum_{\nu=2^{n+1}+1}^{\infty} \sum_{\mu=2^{m+1}+1}^{\infty} \Delta_{l_{1} l_{2}} a_{\mu \nu} \widetilde{B}_{\mu}^{l_{1}}(x) \widetilde{B}_{\nu}^{l_{2}}(y) \chi_{(0, \pi]}(x) \chi_{(0, \pi]}(y)\right]_{y x}^{*}\left(t_{1}, t_{2}\right)\right)^{\alpha} d t_{1} d t_{2} .
\end{aligned}
$$

First we estimate $I_{1}$. Since $\left|\widetilde{B}_{\mu}^{l_{1}}(x)\right| \leq C_{1}(\mu+1)^{l_{1}},\left|\widetilde{B}_{v}^{l_{2}}(y)\right| \leq C_{2}(v+1)^{l_{2}}$, where $C_{1}, C_{2}$ are independent of $x, y$ and $\mu, v$, we have

$$
\begin{aligned}
I_{1} \leq \sum_{n=0}^{\infty} \int_{\pi / 2^{n+4}}^{\pi / 2^{n+3}} \sum_{m=0}^{\infty} \int_{\pi / 2^{m+4}}^{\pi / 2^{m+3}} & \omega\left(16 t_{1}, 16 t_{2}\right) \\
& \left(\sum_{\nu=0}^{2^{n+1}} \sum_{\mu=0}^{2^{m+1}} \Delta_{l_{1} l_{2}} a_{\mu \nu}(\mu+1)^{l_{1}}(\nu+1)^{l_{2}}\right)^{\alpha} d t_{1} d t_{2} .
\end{aligned}
$$

Since $\Delta_{l_{1}-1 l_{2}} a_{m n} \geq 0, \Delta_{l_{1}-1 l_{2}-1} a_{m n} \geq 0$ and $\Delta_{l_{1} l_{2}} a_{m n}=\Delta_{l_{1}-1 l_{2}} a_{m n}-$ $\Delta_{l_{1}-1 l_{2}} a_{m+1 n}, \Delta_{l_{1}-1 l_{2}} a_{m n}=\Delta_{l_{1}-1 l_{2}-1} a_{m n}-\Delta_{l_{1}-1 l_{2}-1} a_{m n+1}$, then

$$
\begin{aligned}
& \sum_{n=0}^{N} \sum_{m=0}^{M} \Delta_{l_{1} l_{2}} a_{m n}(m+1)^{l_{1}}(n+1)^{l_{2}} \\
& \leq C \sum_{n=0}^{N} \sum_{m=0}^{M} \Delta_{l_{1}-1 l_{2}-1} a_{m n}(m+1)^{l_{1}-1}(n+1)^{l_{2}-1}
\end{aligned}
$$


where $C=C\left(l_{1}, l_{2}\right)$. Using this estimate, we get

$$
\begin{aligned}
I_{1} \leq C \sum_{n=0}^{\infty} \int_{\pi / 2^{n+4}}^{\pi / 2^{n+3}} \sum_{m=0}^{\infty} \int_{\pi / 2^{m+4}}^{\pi / 2^{m+3}} \omega\left(16 t_{1}, 16 t_{2}\right) d t_{1} d t_{2} \\
\left(\sum_{\nu=0}^{n} \sum_{\mu=0}^{m} \Delta_{l_{1}-1 l_{2}-1} a_{2^{\mu}-1,2^{\nu}-1} 2^{\mu l_{1}} 2^{\nu l_{2}}\right)^{\alpha} .
\end{aligned}
$$

Now if $\alpha \geq 1$, we use Lemma 3.2 and if $\alpha<1$, we apply Jensen's inequality. Then using (2.1), we write

$$
\begin{aligned}
I_{1} \leq & C \sum_{n=0}^{\infty} \int_{\pi / 2^{n+4}}^{\pi / 2^{n+3}} \sum_{m=0}^{\infty} \int_{\pi / 2^{m+4}}^{\pi / 2^{m+3}} \omega\left(16 t_{1}, 16 t_{2}\right) d t_{1} d t_{2} \\
& \left(\Delta_{l_{1}-1 l_{2}-1} a_{2^{m}-1,2^{n}-1} 2^{m l_{1}} 2^{n l_{2}}\right)^{\alpha} \\
\leq & C \sum_{n=0}^{\infty}(n+1)^{2 \alpha l_{1}} \sum_{m=0}^{\infty}(m+1)^{2 \alpha l_{2}} w_{m n}\left(\Delta_{l_{1}-1 l_{2}-1} a_{m n}\right)^{\alpha}=: C B\left(\alpha, l_{1}, l_{2}\right) .
\end{aligned}
$$

Now we estimate $I_{2}$. We note that for $y \in(0, \pi),\left|\widetilde{B}_{n}^{l_{2}}(y)\right| \leq C y^{-l_{2}}\left(l_{2}=\right.$ $1,2)$, where $C$ is independent of $n$ and $y$. Then by Lemma 3.4, we have

$$
\begin{aligned}
I_{2} \leq & C \sum_{n=0}^{\infty} \sum_{m=0}^{\infty} \int_{\pi / 2^{n+4}}^{\pi / 2^{n+3}} \int_{\pi / 2^{m+4}}^{\pi / 2^{m+3}} \omega\left(16 t_{1}, 16 t_{2}\right) \\
\times & \left.\left(\sum_{\nu=2^{n+1}+1}^{\infty} \sum_{\mu=0}^{2^{m+1}} \Delta_{l_{1} l_{2}} a_{\mu \nu}(\mu+1)^{l_{1}} y^{-l_{2}} \chi_{(0, \pi]}(x) \chi_{(0, \pi]}(y)\right]_{y x}^{*}\left(t_{1}, t_{2}\right)\right)^{\alpha} d t_{1} d t_{2} \\
\leq & C \sum_{n=0}^{\infty} \sum_{m=0}^{\infty} \int_{\pi / 2^{n+4}}^{\pi / 2^{n+3}} \int_{\pi / 2^{m+4}}^{\pi / 2^{m+3}} \omega\left(16 t_{1}, 16 t_{2}\right) d t_{1} d t_{2} \\
& \left(2^{n l_{2}} \sum_{\nu=2^{n+1}+1}^{\infty} \sum_{\mu=0}^{2^{m+1}} \Delta_{l_{1} l_{2}} a_{\mu \nu}(\mu+1)^{l_{1}}\right)^{\alpha} \\
\leq & C \sum_{n=0}^{\infty} \sum_{m=0}^{\infty} \int_{\pi / 2^{n+4}}^{\pi / 2^{n+3}} \int_{\pi / 2^{m+4}}^{\pi / 2^{m+3}} \omega\left(16 t_{1}, 16 t_{2}\right) d t_{1} d t_{2} \\
& \left(2^{n l_{2}} \sum_{\mu=0}^{2^{m+1}} 2^{\mu l_{1}} \Delta_{l_{1}-1 l_{2}-1} a_{\mu, 2^{n+1}+1}\right)^{\alpha} .
\end{aligned}
$$

To verify the last inequality, we use $\sum_{\nu=2^{n+1}+1}^{\infty} \Delta_{l_{1} l_{2}} a_{\mu \nu}=\Delta_{l_{1} l_{2}-1} a_{\mu, 2^{n+1}+1}$ and (4.1). 
Finally, using Lemma 3.2 for $\alpha \geq 1$, and Jensen's inequality for $\alpha<1$, we arrive at $I_{2} \leq C B\left(\alpha, l_{1}, l_{2}\right)$.

In a similar way to the estimates of $I_{1}$ and $I_{2}$, one can also obtain the inequality $I_{3}+I_{4} \leq C B\left(\alpha, l_{1}, l_{2}\right)$, i.e., $I \leq C B\left(\alpha, l_{1}, l_{2}\right)$. Then this gives us inequality (2.2) for $l_{1}=l_{2}=2$, inequality (2.3) for $l_{1}=2, l_{2}=1$, inequality (2.4) for $l_{1}=1, l_{2}=2$, and inequality (2.5) for $l_{1}=l_{2}=1$.

Proof of Theorem 2.2. By Lemma 3.1, the following identities hold a.e.

(A)

$$
\varphi_{1}(x, y)=f_{1}(x, y)=\sum_{n=0}^{\infty} \sum_{m=0}^{\infty} a_{m n} \cos m x \cos n y
$$

$$
=\sum_{n=0}^{\infty} \sum_{m=0}^{\infty} \Delta_{22} b_{m n} \frac{\sin ^{2} \frac{(m+1) x}{2}}{4 \sin ^{2} \frac{x}{2}} \frac{\sin ^{2} \frac{(n+1) y}{2}}{4 \sin ^{2} \frac{y}{2}},
$$

where $b_{m n}=a_{m n}$,

(B)

$$
\begin{aligned}
\varphi_{2}(x, y)=\frac{f_{2}(x, y)+f_{2}(x, \pi-y)}{2} & =\sum_{n=1}^{\infty} \sum_{m=0}^{\infty} a_{m 2 n-1} \cos m x \sin (2 n-1) y \\
& =\sum_{n=1}^{\infty} \sum_{m=0}^{\infty} \Delta_{21} b_{m n} \frac{\sin ^{2} \frac{(m+1) x}{2}}{4 \sin ^{2} \frac{x}{2}} \frac{\sin ^{2} n y}{\sin y},
\end{aligned}
$$

where $b_{m n}=a_{m 2 n-1}$,

(C)

$$
\begin{aligned}
\varphi_{3}(x, y)=\frac{f_{3}(x, y)+f_{3}(\pi-x, y)}{2} & =\sum_{n=0}^{\infty} \sum_{m=1}^{\infty} a_{2 m-1 n} \sin (2 m-1) x \cos n y \\
& =\sum_{n=0}^{\infty} \sum_{m=1}^{\infty} \Delta_{12} b_{m n} \frac{\sin ^{2} m x}{\sin x} \frac{\sin ^{2} \frac{(n+1) y}{2}}{4 \sin ^{2} \frac{y}{2}}
\end{aligned}
$$

where $b_{m n}=a_{2 m-1 n}$,

(D)

$$
\begin{aligned}
\varphi_{4}(x, y) & =\frac{f_{4}(x, y)+f_{4}(\pi-x, y)+f_{4}(x, \pi-y)+f_{4}(\pi-x, \pi-y)}{2} \\
& =\sum_{n=1}^{\infty} \sum_{m=1}^{\infty} a_{2 m-12 n-1} \sin (2 m-1) x \sin (2 n-1) y \\
& =\sum_{n=1}^{\infty} \sum_{m=1}^{\infty} \Delta_{11} b_{m n} \frac{\sin ^{2} m x}{\sin x} \frac{\sin ^{2} n y}{\sin y},
\end{aligned}
$$


where $b_{m n}=a_{2 m-12 n-1}$.

Thus, we have a.e.

$$
\Phi(x, y)=\sum_{n=0}^{\infty} \sum_{m=0}^{\infty} \Delta_{l_{1} l_{2}} b_{m n} \widehat{B}_{m}^{l_{1}}(x) \widehat{B}_{n}^{l_{2}}(y),
$$

where

(A) $\Phi=\varphi_{1}, l_{1}=2, l_{2}=2, \widehat{B}_{m}^{l_{1}}(x)=B_{m}^{2}(x), \widehat{B}_{n}^{l_{2}}(y)=B_{n}^{2}(y)$;

(B) $\Phi=\varphi_{2}, l_{1}=2, l_{2}=1, \widehat{B}_{m}^{l_{1}}(x)=B_{m}^{2}(x), \widehat{B}_{n}^{l_{2}}(y)=\frac{\sin ^{2} n y}{\sin y}$; and $\Delta_{21} b_{m 0}=\Delta_{10} b_{m 0}=b_{m 0}=0, m=0,1,2, \ldots$;

(C) $\Phi=\varphi_{3}, l_{1}=1, l_{2}=2, \widehat{B}_{m}^{l_{1}}(x)=\frac{\sin ^{2} m x}{\sin x}, \widehat{B}_{n}^{l_{2}}(y)=B_{n}^{2}(y)$; and $\Delta_{12} b_{0 n}=\Delta_{01} b_{0 n}=b_{0 n}=0, n=0,1,2, \ldots$;

(D) $\Phi=\varphi_{4}, l_{1}=1, l_{2}=1, \widehat{B}_{m}^{l_{1}}(x)=\frac{\sin ^{2} m x}{\sin x}, \widehat{B}_{n}^{l_{2}}(y)=\frac{\sin ^{2} n y}{\sin y}$ and $\Delta_{11} b_{m 0}=$ $b_{m 0}=0, m=0,1,2, \ldots, \Delta_{11} b_{0 n}=b_{0 n}=0, n=0,1,2, \ldots$

Using Lemmas 3.5 and 3.8, we obtain

$\int_{0}^{2 \pi} \int_{0}^{2 \pi} w\left(t_{1}, t_{2}\right)\left(F_{y x}^{*}\left(t_{1}, t_{2}\right)\right)^{\alpha} d t_{1} d t_{2} \geq \int_{0}^{2 \pi} \int_{0}^{2 \pi} w(x, y)|F(x, y)|^{\alpha} d x d y$,

because of

$$
\left(F_{y x}^{*}\left(t_{1}, t_{2}\right)\right)^{\alpha}=\left(\left((F(x, y))_{y}^{*}\right)^{\alpha}\right)_{x}^{*}\left(t_{1}, t_{2}\right)=\left(|F(x, y)|^{\alpha}\right)_{y x}^{*}\left(t_{1}, t_{2}\right) .
$$

We also note the following:

$$
\begin{aligned}
\int_{0}^{\pi} \int_{0}^{\pi} w\left(\frac{t_{1}}{2}, \frac{t_{2}}{2}\right) w\left(\frac{t_{1}}{2}, \frac{t_{2}}{2}\right)\left[(f(x, y)+f(\pi-x, y))_{y x}^{*}\right]^{\alpha}\left(t_{1}, t_{2}\right) d t_{1} d t_{2} \\
\leq C\left(\int_{0}^{\pi} \int_{0}^{\pi} w\left(\frac{t_{1}}{2}, \frac{t_{2}}{2}\right)\left(|f(x, y)|_{y x}^{*}\left(\frac{t_{1}}{2}, \frac{t_{2}}{2}\right)\right)^{\alpha} d t_{1} d t_{2}\right. \\
\left.\quad+\int_{0}^{\pi} \int_{0}^{\pi} w\left(\frac{t_{1}}{2}, \frac{t_{2}}{2}\right)\left(|f(\pi-x, y)|_{y x}^{*}\left(\frac{t_{1}}{2}, \frac{t_{2}}{2}\right)\right)^{\alpha} d t_{1} d t_{2}\right) \\
\leq C \int_{0}^{2 \pi} \int_{0}^{2 \pi} w\left(t_{1}, t_{2}\right)\left(f_{y x}^{*}\left(t_{1}, t_{2}\right)\right)^{\alpha} d t_{1} d t_{2} .
\end{aligned}
$$

Using this, let us estimate

$$
I:=\int_{0}^{\pi} \int_{0}^{\pi} w\left(\frac{x}{2^{k}}, \frac{y}{2^{k}}\right)|\Phi(x, y)|^{\alpha} d x d y,
$$


where

$$
k:= \begin{cases}0 & \text { if } \Phi=\varphi_{1}, \\ 1 & \text { if } \Phi=\varphi_{2}\left(\text { or } \varphi_{4}\right), . \\ 2 & \text { if } \Phi=\varphi_{3}\end{cases}
$$

We have

$$
\begin{aligned}
I & =\sum_{s_{2}=0}^{\infty} \int_{\pi / 2^{s_{2}}}^{\pi / 2^{s_{2}+1}} \sum_{s_{1}=0}^{\infty} \int_{\pi / 2^{s_{1}}}^{\pi / 2^{s_{1}+1}} w\left(\frac{x}{2^{k}}, \frac{y}{2^{k}}\right)|\Phi(x, y)|^{\alpha} d x d y, \\
& \geq C \sum_{s_{2}=0}^{\infty} \sum_{s_{1}=0}^{\infty} w\left(\frac{\pi}{2^{k+s_{1}}}, \frac{y}{2^{k+s_{2}}}\right) \int_{\pi / 2^{s_{2}}}^{\pi / 2^{s_{2}+1}} \int_{\pi / 2^{s_{1}}}^{\pi / 2^{s_{1}+1}}|\Phi(x, y)|^{\alpha} d x d y \\
& =: C \sum_{s_{2}=0}^{\infty} \sum_{s_{1}=0}^{\infty} w\left(\frac{\pi}{2^{k+s_{1}}}, \frac{y}{2^{k+s_{2}}}\right) A_{s_{1} s_{2}} \geq C \sum_{s_{2}=j}^{\infty} \sum_{s_{1}=i}^{\infty} w\left(\frac{\pi}{2^{k+s_{1}}}, \frac{\pi}{2^{k+s_{2}}}\right) A_{s_{1} s_{2}},
\end{aligned}
$$

where $i=j=0$ for $\varphi_{1}, i=0, j=1$ for $\varphi_{2}, i=1, j=0$ for $\varphi_{3}$, and $i=j=1$ for $\varphi_{4}$.

Using the following estimate (see [16])

$$
A_{s_{1} s_{2}} \geq C 2^{s_{1}\left(l_{1} \alpha-1\right)+s_{2}\left(l_{2} \alpha-1\right)}\left(\Delta_{l_{1}-1 l_{2}-1} b_{\left[2^{s_{1}-1}\right]\left[2^{s_{2}-1}\right]}\right)^{\alpha},
$$

we have

$$
I \geq C \sum_{s_{2}=j}^{\infty} \sum_{s_{1}=i}^{\infty} w\left(\frac{\pi}{2^{k+s_{1}}}, \frac{y}{2^{k+s_{2}}}\right) 2^{s_{1}\left(l_{1} \alpha-1\right)+s_{2}\left(l_{2} \alpha-1\right)}\left(\Delta_{l_{1}-1 l_{2}-1} b_{\left[2^{s_{1}-1}\right]\left[2^{s_{2}-1}\right]}\right)^{\alpha} .
$$

Thus, we get for $i=j=0$

$$
\left\|f_{1}(x, y)\right\|_{\Lambda_{2}^{\alpha}(\omega)} \geq C_{1}\left(\sum_{n=0}^{\infty}(n+1)^{2 \alpha} \sum_{m=0}^{\infty}(m+1)^{2 \alpha} w_{m n}\left(\Delta_{11} a_{m n}\right)^{\alpha}\right)^{\frac{1}{\alpha}} .
$$

In a similar way, one can also obtain inequalities (2.7)-(2.9).

Proof of Theorem 2.3. We only consider in detail the case (A). By Lemma 3.1, one has

$$
f_{1}(x, y)=\sum_{n=0}^{\infty} \sum_{m=0}^{\infty} \Delta_{22} a_{m n} B_{m}^{2}(x) B_{n}^{2}(y)=\sum_{n=0}^{\infty} \sum_{m=0}^{\infty} \Delta_{11} b_{m n} B_{m}^{2}(x) B_{n}^{2}(y),
$$

where

$$
b_{m n}=\Delta_{11} a_{m n}, \quad B_{k}^{2}(z)=\frac{\sin ^{2} \frac{(k+1) z}{2}}{4 \sin ^{2} \frac{z}{2}} .
$$


Let $x \in\left[\frac{\pi}{2^{m+1}}, \frac{\pi}{2^{m}}\right], y \in\left[\frac{\pi}{2^{n+1}}, \frac{\pi}{2^{n}}\right], I_{m n}=\left[\frac{\pi}{2^{m+1}}, \frac{\pi}{2^{m}}\right] \times\left[\frac{\pi}{2^{n+1}}, \frac{\pi}{2^{n}}\right]$, and let

$$
\Psi_{m n}(x, y)=\sum_{\nu=\left[2^{n-1}\right]}^{\infty} \sum_{\mu=\left[2^{m-1}\right]}^{\infty} \Delta_{11} b_{\mu \nu} B_{\mu}^{2}(x) B_{v}^{2}(y) .
$$

Therefore,

$$
f_{1}(x, y) \geq \sum_{n=0}^{\infty} \sum_{m=0}^{\infty} \chi_{I_{m n}}(x, y) \Psi_{m n}(x, y) .
$$

Then by Lemmas 3.5, 3.6, 3.7, and Jensen's inequality, we have

$$
\begin{aligned}
& \left\|f_{1}(x, y)\right\|_{\Lambda_{2}^{\alpha}(\omega)}^{\alpha} \\
& =\int_{0}^{2 \pi} \int_{0}^{2 \pi} \omega\left(t_{1}, t_{2}\right)\left(\left(\left[f_{1}(x, y)\right]_{y}^{*}\right)_{x}^{*}\left(t_{1}, t_{2}\right)\right)^{\alpha} d t_{1} d t_{2} \\
& \geq C \int_{0}^{2 \pi} \int_{0}^{2 \pi} \omega\left(t_{1}, t_{2}\right)\left(\left(\left[\sum_{n=0}^{\infty} \sum_{m=0}^{\infty} \chi_{I_{m n}}(x, y) \Psi_{m n}(x, y)\right]_{y}^{*}\right)_{x}^{*}\left(t_{1}, t_{2}\right)\right)^{\alpha} d t_{1} d t_{2} \\
& =C \int_{0}^{2 \pi} \int_{0}^{2 \pi} \omega\left(t_{1}, t_{2}\right)\left(\left[\left(\sum_{n=0}^{\infty} \sum_{m=0}^{\infty} \chi_{I_{m n}}(x, y) \Psi_{m n}(x, y)\right)^{\alpha}\right]_{y}^{*}\right)_{x}^{*}\left(t_{1}, t_{2}\right) d t_{1} d t_{2} \\
& \geq C \int_{0}^{2 \pi} \int_{0}^{2 \pi} \omega\left(t_{1}, t_{2}\right)\left(\left[\sum_{n=0}^{\infty} \sum_{m=0}^{\infty}\left(\chi_{I_{m n}}(x, y) \Psi_{m n}(x, y)\right)^{\alpha}\right]_{y}^{*}\right)_{x}^{*}\left(t_{1}, t_{2}\right) d t_{1} d t_{2} \\
& \geq C \int_{0}^{2 \pi} \int_{0}^{2 \pi} \omega\left(t_{1}, t_{2}\right) \sum_{n=0}^{\infty} \sum_{m=0}^{\infty}\left(\left[\chi_{I_{m n}}(x, y) \Psi_{m n}(x, y)\right]_{y x}^{*}\left(t_{1}, t_{2}\right)\right)^{\alpha} d t_{1} d t_{2} .
\end{aligned}
$$

We will use the following fact (see [16]): there exists a set $J_{m n} \subset I_{m n}$ such that

$$
J_{m n}=\left\{(x, y) \in I_{m n}: \Psi_{m n}(x, y) \geq C_{1} 2^{2(n+m)} \Delta_{11} b_{\left[2^{m-1}\right]\left[2^{n-1}\right]}\right\}
$$

and $\mu\left(J_{m n}\right) \geq C_{2} \mu\left(I_{m n}\right)$, where $C_{1}, C_{2}$ do not depend on $n, m,\left\{b_{m n}\right\}$. Hence, we have

$$
\begin{aligned}
\left\|f_{1}(x, y)\right\|_{\Lambda_{2}^{\alpha}(\omega)}^{\alpha} & \\
\geq & C \sum_{n=0}^{\infty} \sum_{m=0}^{\infty} \int_{0}^{2 \pi} \int_{0}^{2 \pi} \omega\left(t_{1}, t_{2}\right) \\
& \left(\left(\chi_{I_{m n}}(x, y) 2^{2(n+m)} \Delta_{11} b_{\left[2^{m-1}\right]\left[2^{n-1}\right]}\right)_{y x}^{*}\left(t_{1}, t_{2}\right)\right)^{\alpha} d t_{1} d t_{2} \\
= & C \sum_{n=0}^{\infty} \sum_{m=0}^{\infty} \int_{0}^{2 \pi} \int_{0}^{2 \pi} \omega\left(t_{1}, t_{2}\right)\left(\chi_{T}\left(t_{1}, t_{2}\right) 2^{2(n+m)} \Delta_{11} b_{\left[2^{m-1}\right]\left[2^{n-1}\right]}\right)^{\alpha} d t_{1} d t_{2},
\end{aligned}
$$


where

$$
T=\left\{\left(t_{1}, t_{2}\right):\left(\chi_{I_{m n}}(x, y)\right)_{y x}^{*}\left(t_{1}, t_{2}\right)>\frac{1}{2}\right\} .
$$

By Lemma 3.10, we write

$$
\begin{aligned}
& \left\|f_{1}(x, y)\right\|_{\Lambda_{2}^{\alpha}(\omega)}^{\alpha} \\
& \geq C \sum_{n=0}^{\infty} \sum_{m=0}^{\infty}\left(2^{2(n+m)} \Delta_{11} b_{\left[2^{m-1}\right]\left[2^{n-1}\right]}\right)^{\alpha} \int_{C_{2} \pi 2^{-(n+2)}}^{C_{2} \pi 2^{-(n+1)}} \int_{C_{2} \pi 2^{-(m+2)}}^{C_{2} \pi 2^{-(m+1)}} \omega\left(t_{1}, t_{2}\right) d t_{1} d t_{2} \\
& \geq C \sum_{n=0}^{\infty} \sum_{m=0}^{\infty}\left(2^{2(n+m)} \Delta_{11} b_{\left[2^{m-1}\right]\left[2^{n-1}\right]}\right)^{\alpha} \omega\left(\frac{\pi}{2^{m}}, \frac{\pi}{2^{n}}\right) \\
& \geq C \sum_{n=0}^{\infty}(n+1)^{2 \alpha} \sum_{m=0}^{\infty}(m+1)^{2 \alpha} w_{m n}\left(\Delta_{11} a_{m n}\right)^{\alpha} .
\end{aligned}
$$

In a similar way, one can also obtain inequalities (2.7)-(2.9).

ACKNOWLEDGEMENT. The research was partially funded by Russian Fund of Basic Research (grants no. 06-01-00268 and no. 08-01-00302) and the Leading Scientific Schools (grant NSH-2787.2008.1). The second named author acknowledges support from the Centre de Recerca Matematica (Barcelona) and Scuola Normale Superiore (Pisa).

\section{REFERENCES}

1. Barza, S., Kaminska, A., Persson, L.-E., Soria, J., Mixed norm and multidimensional Lorentz spaces, Positivity 10 (2006), 539-554.

2. Barza, S., Persson, L.-E., Soria, J., Multidimensional rearrangement and Lorentz spaces, Acta Math. Hungar. 104, no. 3 (2004), 203-224.

3. Bennett, C., Sharpley, R., Interpolation of Operators, Academic Press, Boston, MA, 1988.

4. Booton, B., Sagher, Y., Norm inequalities for certain classes of functions and their Fourier transforms, J. Math. Anal. Appl. 335 (2007), no. 2, 1416-1433.

5. Blozinski, A. P., Multivariate rearrangements and Banach function spaces with mixed norms, Trans. Amer. Math. Soc. 263, no. 1 (1981), 149-167.

6. Gulisašvili, A. B., Trigonometric series with monotone decreasing coefficients, and distribution functions, Mat. Zametki 10 (1971), 3-10.

7. Hardy, G. H., On double Fourier series, and especially those which represent the double zetafunction with real and incommensurable parameters, Quart. J. Math. 37 (1905), 53-79.

8. Hardy, G. H., Littlewood, J. S., Notes on the theory of series. XIII: Some new properties of Fourier constants, J. London Math. Soc. 6 (1931), 3-9.

9. Kreı̆n, S. G., Petunīn, Yu. I., Semenov, E. M., Interpolation of linear operators, Amer. Math. Soc. Transl. 54 (1982).

10. Leindler, L., Some inequalities of Hardy-Littlewood type, Anal. Math. 20, no. 2 (1994), 95106. 
11. Miyamoto, T., On sine and cosine series with monotonically decreasing coefficients and generalized Lorentz-Zygmund spaces, Acta Sci. Math. (Szeged) 69, no. 3-4 (2003), 667675.

12. Moricz, F., On double cosine, sine, and Walsh series with monotone coefficients, Proc. Amer. Math. Soc. 109, no. 2 (1990), 417-425.

13. Nursultanov, E. D., On the coefficients of multiple Fourier series from $L_{p}$-spaces, Izv. Ross. Akad. Nauk Ser. Mat. 64, no. 1 (2000), 95-122; translation in Izv. Math. 64, no. 1 (2000), 93-120.

14. Sagher, Y., An application of interpolation theory to Fourier series, Studia Math. 41 (1972), 169-181.

15. Sagher, Y., Interpolation of $r$-Banach spaces, Studia Math. 41 (1972), 45-70.

16. Vukolova, T. M., Dyachenko, M. I., Estimates for the norms of sums of double trigonometric series with multiply monotone coefficients, Russ. Math. 38, no. 7 (1994), 18-26; translation from Izv. Vyssh. Uchebn. Zaved. Mat. 1994, no. 7 (386) (1994), 20-28.

17. Zygmund, A., Trigonometric Series, Vol. I, II, Third edition, Cambridge, 2002.

VOLGOGRAD STATE UNIVERSITY

VOLGOGRAD

400131 RUSSIA

E-mail: htf@vstu.ru
SCUOLA NORMALE SUPERIORE PISA PIAZZA DEI CAVALIERI 7

56126 ITALY

and

INSTITUCIÓ CATALANA DE RECERCA I ESTUDIS AVANCATS

CENTRE DE RECERCA MATEMÀTICA

E-mail: s.tikhonov@sns.it 